\title{
Integration of Microbiota-Gut-Brain Axis: Significance of Autonomic Neural Influence
}

\author{
Gopal Krushna Pal
}

\section{Dr. Gopal Krushna Pal}

Editor-in-Chief, IJCEP, Professor (Senior Scale), Department of Physiology and Programme Director, Advance Center for Yoga, JIPMER, Puducherry, INDIA.

Phone: +91-9344291160

Email: drgkpal@gmail.com

History

- Submission Date: 10-3-2021;

- Review completed: 25-03-2021;

- Accepted Date: 30-03-2021.

DOI : 10.5530/ijcep.2021.8.1.1

Article Available online

http://www.ijcep.org

\section{Copyright}

(c) 2021 Phcog.Net. This is an openaccess article distributed under the terms of the Creative Commons Attribution 4.0 International license.
The Gut-brain Axis (GBA) is the bidirectional communication between the brain and the enteric nervous system of the gut, linking emotional and cognitive centers of the brain with peripheral intestinal functions. Gut microbiota profoundly influences these interactions. The interaction between microbiota and GBA is made bidirectional by signalling from gut-microbiota to brain and from brain to gut-microbiota through neural, endocrine, immune, and humoral communications. Hence, this bidirectional relationship between gut and the brain is more precisely called as microbiota-gut-brain axis (microbiota-GBA). ${ }^{[1]}$ Microbiota-GBA is closely associated with many functions and dysfunctions in health and diseases in human beings. However, most of the data on GBA have been acquired using germ-free animal models, probiotics, antibiotics, and infection studies in experimental models. In clinical medicine, data on functions of microbiotaGBA are derived mainly from the association of dysbiosis with central nervous disorders, especially in autism and anxiety-depressive behaviours and from functional gastrointestinal disorders. Especially, irritable bowel syndrome is considered as a prototype of the disruption of this complex relationship of microbiota-GBA. ${ }^{[2]}$

\section{Organization of Microbiota-GBA}

The Enteric Nervous System (ENS) that consists of the myenteric and mucosal plexuses are regulated by the sympathetic and parasympathetic limbs of the autonomic nervous system (ANS), with the hypothalamic-pituitary-adrenal axis playing a major role in the control of ENS functions. ${ }^{[3]}$ The vagus nerve is the major parasympathetic supply to gut and it impacts alterations in central nervous system (CNS)-responses to changes in the gut environment. Physiologically, the neural vagal pathway is the link between the cognitive and emotional centres of the brain and peripheral intestinal functions. The state of the gut influences the state of the mind of the individual, and much evidence points to the behavioural alterations brought about by the composition of microbiota-GBA. ${ }^{[4]}$ The gut microbiota interacts with various physiological systems and results in modification of many biological functions as well as the outward behaviours. Insights into the gut-brain crosslinkages have revealed a complex communication system that not only ensures the proper maintenance of gastrointestinal homeostasis, but also the homeostasis of many other functions such as immunity, endocrinal functions, stress responses, motivation, and higher cognitive functions. ${ }^{[5]}$

\section{Autonomic Connection of Microbiota- GBA}

The gut-brain bidirectional communication network includes the ANS and the hypothalamic pituitary adrenal (HPA) axis. The ANS with its sympathetic and parasympathetic limbs, drives both afferent signals, arising from the gut lumen and transmitted though ENS-vagal pathways to CNS, and efferent signals from CNS to the intestinal wall. The HPA axis is considered as the core stress efferent axis of the body that coordinates the adaptive responses of the organism to stressors of any kind. HPA closely interacts with the limbic system, which is predominantly involved in the control of visceral functions, memory and emotional responses. ${ }^{[4]}$ In the HPA axis, the corticotropin-releasing factor from the hypothalamus stimulates the secretion of adrenocorticotropic hormone from pituitary gland, which in turn influences release of cortisol from the adrenal glands. Cortisol is a major hormone that mediates stress responses and controls functions of many organs of the body. The functions of HPA axis including the modulation of cortisol functions are strongly influenced by limbic system, in which vagus nerve plays a critical role in the integration of stress mediated inflammatory responses. Therefore, vagus nerve has been proposed to be the key integrator of inflammatory reflex. ${ }^{[6]}$ Thus, both neural and hormonal pathways of communication combine to allow brain to influence the activities of the gut including functions of immune cells, epithelial cells, enteric neurons, smooth muscle cells, interstitial cells of Cajal and enterochromaffin cells. These cells in the gut profoundly influence the gut microbiota, and gut microbiomes in turn send afferent signals to the brain mainly via vagus nerve as part of reciprocal communication of gut-brain interaction. Thus, the gut microbiota plays an important role in bidirectional interactions between the gut and the nervous system in the regulation of neuro-endocrine systems associated with stress response, anxiety and memory function, in which ANS appears to be the key mediator. Therefore, the communication network 
between the gut and the CNS that includes the ENS, sympathetic and parasympathetic branches of ANS, neuroendocrine signalling pathways including HPA axis, and neuroimmune systems is thus the target of therapy in gut-dysbiosis related disorders. ${ }^{[2]}$

\section{Mechanism of Autonomic Influence on Microbiota-GBA}

Afferent signals from GI tract and vagal sensory neurons from the gut carry visceral feedback from the gut structures to the thoracic and upper lumbar spinal cord and to the nucleus tractus solitarius located in the caudal brainstem, engaging polysynaptic inputs to higher brain regions, including the hypothalamus and limbic forebrain. Bi-directional control is provided by descending pre-autonomic neural projections from the cingulate and insular cortices, amygdala, bed nucleus of the stria terminalis, and hypothalamus, all of which are positioned to alter vagal and spinal autonomic outflow to the gut. ${ }^{[3]}$ Thus, the microbiota-GBA has the both components of ANS in its afferent and efferent connections, in which vagal fibers predominate in the linking mechanisms. The parasympathetic component of ANS has been suggested to mediate as the most important neural pathway for bidirectional link between gut microbes and the brain. In this interaction, the vagus nerve appears to be necessary for several gut functions induced by two separate probiotic strains in rodents. ${ }^{[4]}$ Specifically, treatment with Lactobacillus rhamnosus (JB-1) led to region-dependent alterations in central GABA receptor expression, accompanied by reduced anxiety- and depression-like behaviour and attenuation of stress-induced cortisol response, and all these ameliorating effects of probiotic treatment were dependant on the intact vagus nerve. ${ }^{[1]}$ Also, the anxiolytic effect of Bifidobacterium longum was absent in vagotomised mice, in a colitis model.

\section{Future Perspective}

Though additional signalling systems, in addition to ANS mechanisms are likely to be involved in microbiota-brain-gut communication, vagal pathway appears to be the primary interlinking-facilitator. As vagus nerve activity is profoundly influenced by yoga, especially by slow breathing exercises, the future studies should focus on the role on slow pranayama as a therapy for gut dysbiosis-induced clinical disorders.

\section{REFERENCES}

1. Carabotti M, Scirocco A, Maselli MA, Severi C. The gut-brain axis: interactions between enteric microbiota, central and enteric nervous systems. Ann Gastroenterol. 2015;28(2):203-9.

2. Chandran S, Manohari SM, Raman V. The gut-brain connection: A qualitative review of the conceptualisation and implications of the gut-brain-microbiome axis. Tel J Psychiatr. 2019;5(2):94-103.

3. Dinan TG, Cryan JF. Regulation of the stress response by the gut microbiota: Implications for psychoneuroendocrinology. Psychoneuroendocrinology 2012;37(9):1369-78

4. Lucas G. Gut thinking: The gut microbiome and mental health beyond the head. Microb Ecol Health Dis. 2018;29(2):1548250. https://doi.org/10.1080/ 16512235.2018.

5. Foster JA, Rinaman L, Cryan FJ. Stress and the gut-brain axis: Regulation by the microbiome. Neurobiology of Stress 2017;7:124-36.

6. Pal GK, Nanda N. Vagus nerve: The key integrator of anti-inflammatory reflex. Int J Clin Exp Physiol. 2020;7(1):1-2

Cite this article: Pal GK. Integration of Microbiota-Gut-Brain Axis: Significance of Autonomic Neural Influence. Int J Clin Exp Physiol. $2021 ; 8(1): 1-2$ 